\title{
La formación académica de los jóvenes y las pruebas de admisión a la educación superior. Una experiencia peruana
}

\author{
The academic training of young people and the admission tests to higher education: $A$ \\ Peruvian experience
}

\section{A formação acadêmica dos jovens e os testes de admissão ao ensino superior. Uma experiência peruana}

\author{
Rómulo Cresenciano Romero Centeno \\ rrecente@gmail.com; romercenteno@gmail.com \\ https://orcid.org/0000-0001-9883-0237 \\ Universidad Nacional de Ingeniería, Lima-Perú
}

Recibido 22 de abril 2021 | Arbitrado y aceptado 15 de mayo 2021 | Publicado en 01 julio 2021

\section{RESUMEN}

El objetivo de esta investigación se basa en valorar la influencia de la formación precedente en los estudiantes peruanos de los centros preuniversitarios de universidades estatales para lograr el éxito en las pruebas de acceso a las instituciones de la educación superior. La metodología aplicada se basa en un diseño bibliográfico de tipo documental, de la misma manera esta investigación se apoya en un diseño no experimental de tipo de campo con nivel descriptivo y explicativo. La educación preuniversitaria no forma parte del sistema educativo peruano; pero, para ingresar a la universidad nacional, es una estación obligatoria. La investigación concluye que la educación básica regular estatal no prepara a los estudiantes para seguir estudios de educación superior porque los cinco cursos que brinda en 11 años de educación no son suficientes para responder los complejos exámenes de admisión de cualquier universidad nacional.

Palabras-clave: Admisión; Estudio preuniversitario; Colegio nacional; Rendimiento académico

\section{ABSTRACT}

The objective of this research is based on assessing the influence of the previous training on Peruvian students from pre-university centers of state universities to achieve success in entrance exams to higher education institutions. The applied methodology is based on a documentary-type bibliographic design, in the same way this research is supported by a nonexperimental field-type design with a descriptive and explanatory level. Pre-university education is not part of the Peruvian educational system; but, to enter the national university, it is a mandatory station. The research concludes that state regular basic education does not prepare students for higher education studies because the five courses it provides in 11 years of education are not enough to answer the complex entrance exams of any national university.

Key words: Admission; Pre-university study; National college; Academic performance

\section{RESUMO}

O objetivo desta pesquisa baseia-se em avaliar a influência da educação anterior nos estudantes peruanos dos centros pré-universitários das universidades estaduais para obter sucesso nos exames de admissão às instituições de ensino superior. A metodologia aplicada é baseada em um desenho bibliográfico de tipo documental, da mesma forma que esta pesquisa é baseada em um desenho não experimental de tipo de campo com nível descritivo e explicativo. A educação pré-universitária não faz parte do sistema educacional peruano, mas é uma estação obrigatória para a entrada na universidade nacional. A pesquisa conclui que a educação básica regular do estado não prepara os estudantes para prosseguir estudos superiores porque os cinco cursos que oferece em 11 anos de educação não são suficientes para responder aos complexos exames de admissão de qualquer universidade nacional.

Palavras-chave: Admissão; Estudo pré-universitário; Faculdade nacional; Desempenho acadêmico; Desempenho acadêmico 


\section{INTRODUCCIÓN}

El desarrollo de la educación superior en un país constituye un espacio para la formación de las futuras generaciones, de ella depende en alguna medida su crecimiento. Los jóvenes profesionales formados en este nivel se constituirán en la principal fuerza laboral que moverá las industrias, la economía, la tecnología, y guiará al país en los próximos años, como los líderes que tendrán cierto impacto e influencias para promover los cambios y transformaciones de la sociedad. Llegar a este nivel y lograr su acceso es un proceso complejo que afecta a una parte importante de la población juvenil que aspira a su formación profesional universitaria.

La educación superior en la región ha tenido en las últimas décadas determinadas trasformaciones, dentro del marco de políticas educativas que favorecen la diversidad de las instituciones, cambios en los enfoques curriculares dirigidos a satisfacer las exigencias del mercado laboral. Según Didriksson (2020), las tendencias de cambio que ocurren a nivel de la educación superior están basadas esencialmente en la relación de la universidad con la economía considerando la relación del Estado con el mercado.

Uno de los factores que influyen en el acceso a la educación superior es la trayectoria académica de los estudiantes durante el nivel precedente. En la región, los niveles precedentes a los estudios superiores están cejados por un conjunto de situaciones que van desde el analfabetismo, insuficiente cobertura escolar, baja calidad en la formación, entre otros, que profundizan las situaciones de pobreza, desigualdad y exclusión, dejando un sector de los adolescente y jóvenes sin la posibilidad de acceder a nivel de educación superior (Miranda, 2018).

En este contexto, la Superintendencia Nacional de Educación Superior Universitaria (2021) en el Perú, menciona que se cuenta en el país con alrededor de 140 universidades, sin embargo solo 94 universidades están licenciadas, aunque según
Bravo (2018), el 70\% de los jóvenes egresados de secundaria no va a la educación superior, asimismo Maza (2019) del diario el Comercio menciona que el Ministro de Educación Daniel Alfaro en el marco del foro Competitividad y Productividad indicó que 6 de cada 10 jóvenes peruanos no acceden a educación superior. Otros testimonios que demuestra la situación de la educación peruana, según datos de la Secretaria Nacional de la Juventud (2019) la población de jóvenes representaba el 25\% de la población peruana lo que se traduce a cerca de 8 millones de personas de esta población solo un $21 \%$ se dedica a sus estudios.

Una de las dificultades para el acceso a este nivel de estudios (universitario) son las pruebas de admisión o de ingreso, comprobaciones que se realizan al finalizar la educación secundaria y que evalúan un sistema de conocimientos y habilidades perteneciente a diferentes disciplinas que los estudiantes deben haber obtenido en el nivel precedente al nivel de educación superior. Estas pruebas están dirigidas a medir esencialmente conocimientos de forma casi memorística; es decir, el acceso a una universidad nacional solo depende de dar un buen examen de admisión y para ello, el postulante debe haber adquirido en los años precedentes mucho conocimiento (Angulo, Angulo, Huamán, y Espino, 2016)

Estas comprobaciones o exámenes de admisión ocurren en un momento determinado (dos veces al año en la mayoría de universidades nacionales). En muchas ocasiones, los estudiantes se preparan con antelación para tener éxito en su ejecución, aun así, no todos logran buenos resultados. Según investigaciones recientes Romero (2020), uno de los factores que incide en los malos resultados es la formación que reciben los estudiantes en los años precedentes. Al revisar la malla curricular de las instituciones públicas y el balotario (conjunto de temas de cada asignatura) de admisión de cada universidad nacional, el nivel de exigencias 
en cuanto a los conocimientos y habilidades que requieren estas últimas, es amplia; lo que demuestra la falta de congruencia entre la formación recibida y las exigencias de las pruebas de admisión.

La formación de los adolescentes y jóvenes para continuar los estudios profesionales de nivel medio o superior es un proceso en el cual inciden diferentes factores, aunque en el artículo se prioriza la formación académica que brinda los centros preuniversitarios, en el contexto de la educación peruana. Los jóvenes en la ciudad tienen la opción de educarse en colegios estatales o colegios privados. Los primeros se rigen por la malla curricular y la dirección del Ministerio de Educación; los segundos, no trabajan con mallas curriculares dirigidas desde el Ministerio. La malla curricular que utilizan está, esencialmente, enfocada a los prospectos de los exámenes de admisión de las universidades nacionales.

La educación preuniversitaria, según Hurtado (2006), es la que "realiza una persona antes de presentarse a un concurso de admisión, generalmente de alguna universidad". Esta preparación fue vista por muchos como el "puente necesario entre el colegio y la universidad” (p. 168). Hurtado añade que la preparación preuniversitaria "ya no es posterior al término de la secundaria, sino durante esta; los profesores preuniversitarios han pasado a formar parte de la plana docente de los colegios, y muchos profesores de carrera han adaptado su trabajo al tipo preuniversitario" (p. 168).

Para otras realidades educativas, la educación preuniversitaria se define y tiene objetivos específicos en el sistema educativo como se constató.

En Brasil, se le conoce como educación vocacional o técnica. No es obligatoria y sus principales objetivos son la preparación académica, ciudadanía y preparación para el empleo (Núñez, 2013).
En Chile, se le denomina Educación Media. Es obligatoria y tiene dos modalidades: humanísticocientífica y técnico-profesional. En esta última, los estudiantes obtienen los títulos de licenciado en educación media y de técnico de nivel medio en la especialidad que hayan cursado (Núñez, 2013). Aunque (Gonzales, 2016) considera a la educación preuniversitaria como un sistema informal, de lucro, e indispensable para que los alumnos se nivelen y superen los exámenes de admisión de las universidades.

En España, se le denomina Bachillerato y forma parte de la educación postobligatoria. Se divide en régimen general y especial. Al concluir estos estudios, el estudiante recibe el título de bachiller, que lo faculta para continuar la educación superior (Núñez, 2013, p. 16).

En México, la educación media-superior, o bachillerato, complementa la enseñanza primaria superior, que capacita a los alumnos para vivir útilmente en la nación, y prepara a los alumnos para el ingreso a la universidad (Quiles y Zaragoza, 2020).

En Cuba, también se le denomina Educación preuniversitaria. Abarca del décimo al duodécimo grado, donde los estudiantes profundizan conocimientos y habilidades para continuar con los estudios universitarios. La educación de nivel medio superior está orientada a la formación de los adolescentes de 15 a 17 años. Se ofrece educación nivel medio superior en Institutos Preuniversitarios Vocacionales de Ciencias Exactas (Unesco , 2020).

Es evidente que en estos países la educación preuniversitaria está contemplada y estructurada por el Estado mediante los ministerios de educación. Esta, no reemplaza a la educación básica, sino que es un nivel diferente y complementario, e incluso, en algunos casos obligatoria. Tiene el objetivo de preparar al estudiante para los estudios superiores; sin embargo, no se trata solo de una preparación 
académica, incluye también el desarrollo de habilidades en diferentes aspectos y la orientación vocacional de los estudiantes.

En este sentido el objetivo de esta investigación se basó en valorar la influencia de la formación precedente en los estudiantes peruanos de los centros preuniversitarios de universidades estatales para lograr el éxito en las pruebas de acceso a las instituciones de la educación superior.

\section{MÉTODO}

Esta investigación posee un diseño bibliográfico de tipo documental, pues se basa en la revisión sistemática, rigurosa y profunda de material documental, donde se realiza el análisis de diversos fenómenos o se establece la relación entre dos o más variables, a su vez se mención que es documental pues se recopiló información de diversas fuentes para analizar los datos provenientes de ellos (Palella Stracuzzi y Martins Pestana, 2010).

De la misma manera esta investigación se apoya en un diseño no experimental de tipo de campo con nivel descriptivo y explicativo que según (Palella Stracuzzi y Martins Pestana, 2010) es no experimental ya que se realiza sin manipular de forma deliberada ninguna variable, igualmente es de campo pues se recolectan datos directamente del lugar donde ocurren los hechos que permite describir e interpretar los hechos a través de registros y análisis e interpretaion de la naturaleza actual, e igualmente explicativo puesto que se centra en determinar el origen o las causas de una serie de fenómenos complejos y delicados.

\section{Población y muestra}

La investigación se desarrolló durante ciclo 2020-1 en el Centro de Estudios Preuniversitario de la Universidad Nacional de Ingeniería (Cepreuni), Lima-Perú, que comprende desde el mes de septiembre de 2019 a febrero 2020. Tuvo como población de 2700 estudiantes, en mayor cantidad de la ciudad de Lima. Se ha considerado una muestra de 401 jóvenes de 16 a más 22 años de edad como se muestra en la Tabla 1.

Tabla 1. Edades de los estudiantes Cepreuni, febrero 2020.

Edades de los estudiantes Cepreuni, febrero 2020

\begin{tabular}{ccc}
\hline $14-17$ & 137 \\
$18-21$ & 259 \\
$22+$ & 5 \\
Total & $\mathbf{4 0 1}$ \\
\hline
\end{tabular}

\section{Puntos a considerar}

Para el desarrollo de la investigación, se selecciona como referencia las instituciones Universidad Nacional Mayor de San Marcos (UNMSM) y Universidad Nacional de Ingeniería (UNI). Se realizó un estudio comparado entre las mallas curriculares de las dos instituciones y el del Ministerio de Educación con el propósito de constatar las diferencias en los cursos. Los pocos cursos que llevan en la educación secundaria y los muchos cursos que se consideran en el examen de admisión.

Posteriormente se aplica una encuesta demográfica, validada para esta investigación por juicio de expertos, que permitió determinar la procedencia de colegio estatal o privado, las veces o ciclos que se prepara para postular a la universidad y se realizó un análisis de las diez evaluaciones (7 
prácticas calificadas y 3 exámenes) que se evalúan en las veinte semanas de clases en el centro de estudios preuniversitarios.

\section{RESULTADOS Y DISCUSIÓN}

En el caso de Perú, según la malla curricular de Educación Básica Regular del ministerio de
Educación (2017), los niños, niñas y adolescentes pasan por el proceso educativo de acuerdo con su evolución física, afectiva y cognitiva, desde el momento de su nacimiento. Esta modalidad se organiza en tres niveles como se muestra en la Tabla 2.

Tabla 2. Grados de estudio en Educación Básica en Perú.

\begin{tabular}{|c|c|c|c|c|c|c|c|c|c|c|c|c|c|}
\hline \multirow{3}{*}{$\begin{array}{c}\text { NIVEL } \\
\text { Ciclos } \\
\text { Grados/edades }\end{array}$} & \multicolumn{2}{|c|}{ EDUCACIÓN INICIAL } & \multicolumn{6}{|c|}{ EDUCACIÓN PRIMARIA } & \multicolumn{5}{|c|}{ EDUCACIÓN SECUNDARIA } \\
\hline & I & II & & & & & & & & & & VII & \\
\hline & $0-2$ AÑOS & 3-5 AÑOS & $1^{\circ}$ & $2^{\circ}$ & $3^{\circ}$ & $4^{\circ}$ & $5^{\circ}$ & $6^{\circ}$ & $1^{\circ}$ & $2^{\circ}$ & $3^{\circ}$ & $4^{\circ}$ & $5^{\circ}$ \\
\hline
\end{tabular}

Fuente: (Ministerio de Educación, 2017).

Según Piscoya (2004), el Diseño Curricular Nacional condena a las disciplinas científicas, pues considera que este plan de estudios es muy particular y carece de símil en todos los países desarrollados y de mediano desarrollo, es que en él aparece como única disciplina pasible de enseñanza especializada la Matemática. Entonces, los adolescentes no tienen que estudiar específicamente Física, Química, Biología, Lógica, Psicología, Economía Política, Historia del Perú, Ecología, Informática, como ocurre en otros países. En esta misma línea de análisis, Neira (2020) sostiene que desde hace 30 años el Perú se encuentra educando por áreas, ha desaparecido la enseñanza por materias. Antes se enseñaba ciencias y humanidades y ha desaparecido. El joven peruano sale de secundaria sin oficio y sin formación para ir a estudios superiores.

Al concluir y aprobar los estudios de secundaria, los jóvenes deben postular para continuar los estudios superiores en la universidad nacional. El costo de admisión varía según cada universidad. Por lo general, los estudiantes que concluyen secundaria deben pasar por la preparación en alguna academia o recurrir a los Centros de Estudios Preuniversitarios de la universidad que tiene previsto postular. Para estudiar en estos centros preuniversitarios debe pagar la pensión mensual de 500 soles por un periodo de 5 meses, aproximadamente.

El estudio en un centro de preparación preuniversitario, así sea de la universidad, no forma parte del sistema de educación del Perú, es informal, pero necesario para adquirir los conocimientos que se requieren para ingresar a una universidad nacional. Es decir, quien no puede solventar la mensualidad de los centros preuniversitarios, tiene poquísima probabilidad de seguir los estudios en alguna universidad nacional.

\section{Factores que inciden en el rendimiento escolar de adolescentes y jóvenes}

El rendimiento escolar, según su origen etimológico, rendimiento proviene del latín 'reddere' [re (hacia atrás) y dare (dar)], que luego -por la influencia de prehendere (prender) y vendere (vender)- pasó a ser 'rendere', así lo analiza Flores (2010); por lo que, se entiende una medida de proporción que aparece entre las formas usadas para obtener algo y evaluar el resultado que concretamente se consigue (Morazán, 2013). 
Según Lamas (2015), la complejidad del significado de rendimiento académico se inicia en la conceptualización; es decir, hay diversas denominaciones como aptitud escolar, desempeño académico o rendimiento escolar, aunque, convencionalmente se ha determinado que rendimiento académico se debe usar en poblaciones universitarias y rendimiento escolar en poblaciones de educación básica regular y alternativa.

Para Mandelman, Barbot, y Grigorenko (2016), también, el rendimiento académico ha estado asociado más a la educación básica y muy pocas a la educación superior. El propósito del rendimiento escolar o académico, es alcanzar una meta educativa, un aprendizaje, de igual manera, (Lamas, 2015; Castrillón, Sarache, y Ruiz-Herrera, 2020), los factores más influyentes en el desempeño académico o rendimiento escolar son la preparación pedagógica de los profesores y su calidad académica, los horarios de clase adecuados, la buena relación docente-estudiante y la formación recibida con anterioridad manifestada en la malla curricular que la sustenta.

Según investigaciones realizadas por el Instituto Peruano de Economía-IPE (2018), el rendimiento escolar depende de dos factores; el primero hace referencia a características intrínsecas del estudiante como los hábitos y aptitudes de estudio, el deseo de superación, las condiciones socioeconómicas y el contexto familiar. El segundo factor guarda relación con características que son ajenas al estudiante como la infraestructura del centro de estudios, la malla curricular a desarrollarse, la plana docente y depende de la adecuada implementación y ejecución de las políticas públicas.

Esta situación, hace que los estudiantes que accederán a la educación superior apenas son un número significativo de toda la población con edad para hacerlo, si lo anterior se le suma que la preparación recibida en las instituciones escolares para los que logran quedarse en ellas, no obtienen aprendizajes ni desarrollan las competencias necesarias para el desarrollo de una vida exitosa y su eventual incorporación al mundo del trabajo Miranda (2018), lograr un acceso a la educación superior es un proceso de alta complejidad.

\section{Las pruebas de admisión o ingreso en la realidad peruana}

En el Perú, las condiciones y requisitos de acceso al nivel de educación superior están dadas por el tipo de institución a la que postula; es decir, cada institución cuenta con la autonomía para decidir qué método emplear en la selección de sus ingresantes.

El examen de admisión de una universidad varía según sean la institución privada o estatal. La primera, en su mayoría, requiere solo de una economía familiar solvente; la segunda somete a un proceso de selección para las pocas vacantes que ofrecen. El examen de admisión ordinario se encarga de poner a prueba los conocimientos y habilidades adquiridas en la educación básica, estos exámenes son semejantes de una institución a otra. Por ejemplo, en la Universidad Nacional Mayor de San Marcos (UNMSM) proponen 100 preguntas con el examen tipo Deco y de cinco tipos; es decir para cada área (A, B, C, D y E); en la Universidad Nacional de Ingeniería (UNI) deben aprobar y obtener los mejores puntajes en los tres exámenes desarrollados en tres días no consecutivos, cuyos cuestionarios son con preguntas de respuestas múltiples. Los temas abordados en ellos se clasifican en aptitudes y conocimientos.

En el primer caso, se basan en el razonamiento verbal y matemático; mientras que, en el segundo caso que podemos dividir, por comprensión en dos, el primero evalúa temas de matemática y el segundo, evalúa las ciencias formales. En la Universidad Nacional de Ingeniería, deben aprobar y obtener los mejores puntajes en los tres exámenes desarrollados en tres días no consecutivos, cuyos cuestionarios son con preguntas de respuestas múltiples. 
Otra de las alternativas de ingreso al nivel de educación superior en el Perú, especialmente de las estatales, está destinada a los mejores promedios, el $1 .^{\circ}$ y el $2 .^{\circ}$ de la escuela secundaria; aunque si hay más postulantes que las vacantes ofrecidas por la universidad nacional también deben rendir el examen de admisión. A quienes no obtuvieron los primeros puestos, el camino les resulta más sinuoso para obtener una de las pocas vacantes de la universidad estatal, tanto para los que proceden de escuelas privadas como públicas.

\section{Indagaciones exploratorias}

La investigación se sustenta en otras indagaciones empíricas realizadas en el país como es la Encuesta Censal de Estudiantes (ECE, 2014) del ministerio de Educación a 1228 personas. En esta indagación, se demostró que en la parte de conocimientos están en promedio igual los colegios privados y colegios estatales. Ya en el 2013, se encontró que en 31 de 43 distritos de Lima Metropolitana, los alumnos de colegios públicos tenían un rendimiento superior en matemática al de los privados. Esta realidad se repitió en todos los distritos del Callao. Sin embargo, estos resultados comparados con los promedios internacionales o el rápido acceso a una universidad nacional es una ilusión porque en exámenes internacionales se sigue cerrando la cola de países latinoamericanos; asimismo, en los concursos de admisión a universidades nacionales no son suficientes ya que solo han evaluado dos cursos: Matemática y Comprensión de Lectura.

Según Asencios (2016), los factores que afectan el rendimiento de los alumnos en el Perú están asociados a la oferta educativa, como las características del colegio, el nivel de exigencia de los docentes, la infraestructura escolar, entre otras. El otro factor a tomar en cuenta está asociado a la demanda educativa, como las características del estudiante, la lengua materna, sexo, asistencia a la educación inicial, edad, entre otros. Además, están los asociados a la familia, como las características del hogar (luz, agua, desagüe en casa, número de miembros, disposición decapital o zona rural, entre otras).

\section{El análisis comparativo}

En la Tabla 3, se muestran los cursos de cada nivel sobre lo que brinda el Ministerio de Educación y los cursos que evalúa para el acceso a la universidad las instituciones estatales en mención. La diferencia es que el Estado en la educación básica solo le brinda 5 cursos y les deja sin opción a ingresar a las universidad del Estado porque hay cursos en los que no los ha formado. 
Tabla 3. Estudio comparativo de las Mallas curriculares de los cursos de Educación básica y los cursos evaluados en examen de admisión de dos universidades nacionales de Lima-Perú.

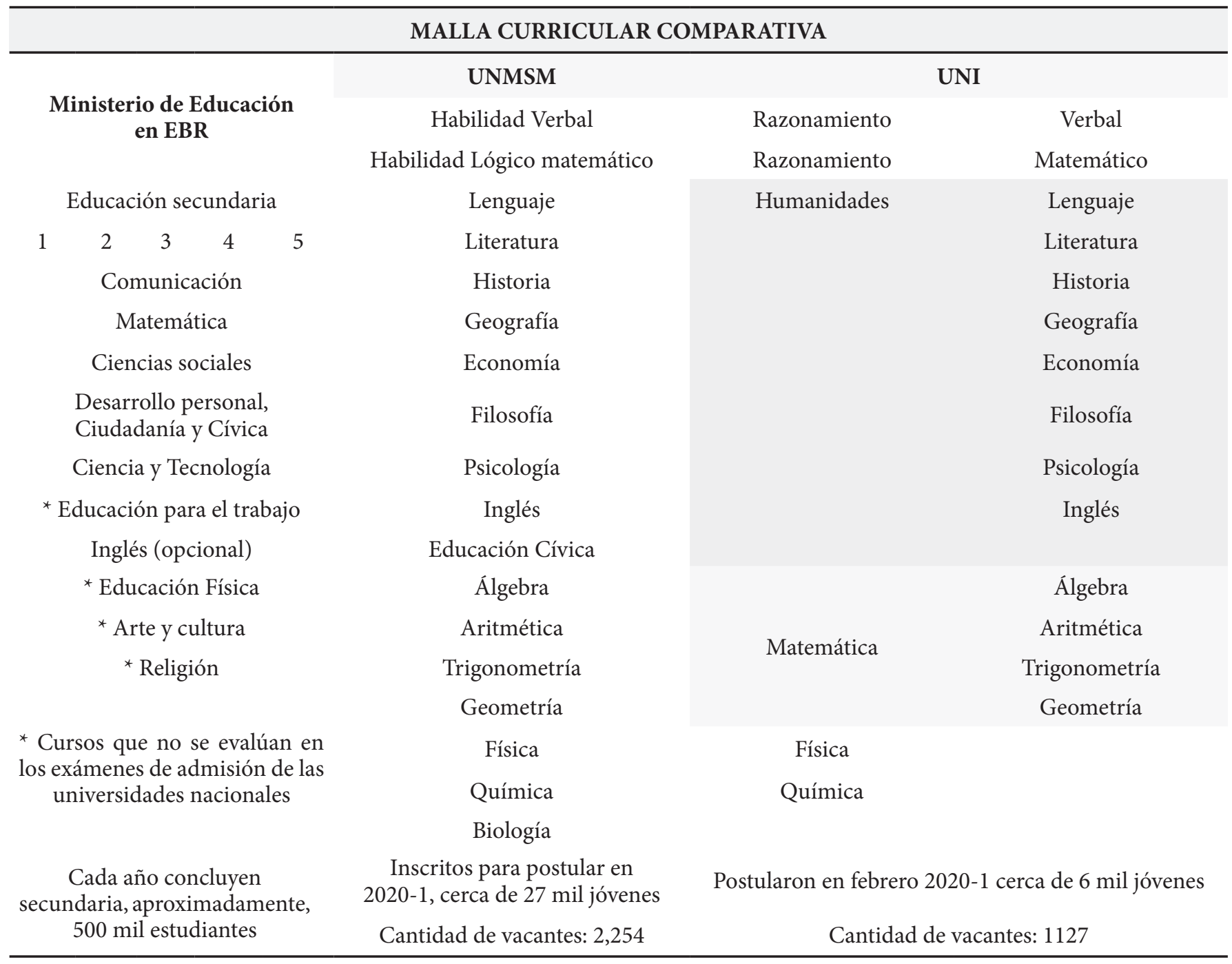

Fuente: (Romero, 2021).

Como se puede apreciar, los cinco cursos enseñados en secundaria estatal (objetivo del Minedu) resultan insuficientes para postular. Hay asignaturas que no se dictan en la secundaria como Economía, Psicología, Filosofía, Química, Física, Biología, Literatura, Geografía, Habilidad o Razonamiento Verbal y Matemático, en matemática se ahonda en Trigonometría, Geometría, Álgebra, Aritmética. Esta disimilitud entre los sílabos de los prospectos de admisión de las universidades estatales y la malla curricular de centros educativos del Estado ponen el primer obstáculo. Los sílabos de admisión presentan cursos como Filosofía, Psicología, Economía, Literatura, Biología, Química, Física, para el examen de admisión. En tanto, en la malla del ministerio de Educación solo aparecen como temas incluidos en la transversalización.

En cuanto a colegios privados, hay mayor libertad de desarrollar los programas educativos según las universidades a las cuales pretende que sus clientes-estudiantes postulen; es decir, desarrollan los cursos según el sílabo de las universidades a la cual pretenden que sus egresados postulen. Estos últimos, a pesar de seguir el temario de los 
sílabos de admisión desarrollados, también, asisten a los Centros de Estudios Preuniversitarios para lograr una plaza en el concurso de admisión de la universidad.

Los jóvenes con aspiraciones universitaria, luego de concluir la secundaria o incluso antes, asisten al ciclo de preparación que consiste en una formación acelerada de cada asignatura que permite a los estudiantes completar los conocimientos o adquirir conocimientos de los cursos que no trataron en la secundaria. Adicionalmente, esta preparación del joven alumno permitirá familiarizarse con las mallas curriculares de su futura carrera. Le permitirá el ansiado ingreso a la universidad.

En los centros de estudio preuniversitario también hay la modalidad del ingreso directo y la modalidad por admisión. Los primeros solo necesitan obtener los primeros puestos, es decir, los primeros puestos para cada carrera; los segundos, deben ocupar la vacante ofertada en el examen de admisión programado. En el caso del ingreso directo, los postulantes a la Universidad Nacional de Ingeniería deberán sacarlas mejores notas en 10 exámenes progresivos.

En resumen, la educación básica, como está organizado por el ministerio de Educación, no provee del conocimiento suficiente para poder ingresar a la universidad por medio del examen de admisión, lo cual implica que el aspirante a universitario tenga que llevar a cabo estudios preuniversitarios que le va a dar mayores conocimientos para ingresar. Esta situación de competencia y nivel de conocimiento le genera a él y la familia una carga de exigencia, de responsabilidad, de organización de tiempo y ocio, de gasto económico, lo cual muchas veces si el alumno no está sólidamente decidido a forjarse una profesión y cubrir sus expectativas puede convertirse en un escollo.

Posterior a la aplicación de la encuesta se procedió a recopilar la información y a reflejar los datos a través de tablas y se muestran a continuación.

En la Tabla 4 se muestran una serie de generalidades sobre la edad de los estudiantes del Cepreuni, Febrero 2020.

Tabla 4. Edades de los estudiantes Cepreuni, febrero 2020.

\begin{tabular}{ccc}
\hline \multicolumn{3}{c}{ Edades de los estudiantes Cepreuni, febrero 2020 } \\
\hline $14-17$ & 137 & $34.16 \%$ \\
$18-21$ & 259 & $64.59 \%$ \\
$22+$ & 5 & $1.25 \%$ \\
Total & $\mathbf{4 0 1}$ & $\mathbf{1 0 0} \%$ \\
\hline
\end{tabular}

En esta Tabla 4 se observa que los estudiantes que se van preparando desde el tercer grado de secundaria sus edades oscilan entre 14 y 17 años la cual es la edad para postularse aquellos que desean ingresar a una universidad nacional y esto equivale al $34,16 \%$, sin embargo se nota que existe un incremento de jóvenes entre 18-21 cursando estos estudios preuniversitarios con un $64,59 \%$ lo que permite deducir que muchos de los jóvenes entre 14-17 años no logran pasar las pruebas de ingreso por lo que siguen durante años en la preparación para lograr un cupo.

Seguidamente en la Tabla 5 se muestra cuantas veces se han preparado los jóvenes en los cursos preuniversitarios. 
Tabla 5. Veces de preparación.

\begin{tabular}{ccc}
\hline CICLOS & CANTIDAD & FRECUENCIA \\
\hline Pocos ciclos & 221 & $55.11 \%$ \\
Regulares ciclos & 170 & $42.39 \%$ \\
Demasiados ciclos & 10 & $2.49 \%$ \\
Total & $\mathbf{4 0 1}$ & $\mathbf{1 0 0 \%}$ \\
\hline
\end{tabular}

En la Tabla 5, se aprecia la cantidad de ciclos de preparación. Pocos ciclos, que significa uno a tres ciclos, el 55.11\%; regulares ciclos, que para el caso significa de 4 a 6 ciclos, un $42.39 \%$; y demasiados ciclos que implica más de 7 ciclos un $2.49 \%$.
De la misma manera en la Tabla 6 se muestra de que colegio provienen los jóvenes que se encuentran en el curso preuniversitario.

Tabla 6. Colegio donde estudio secundaria.

\begin{tabular}{ccc}
\hline Colegio secundaria & Nro de estudiantes & Porcentaje \\
\hline Colegio estatal & 132 & $32.92 \%$ \\
Más en estatal que en particular & 24 & $5.99 \%$ \\
Más en particular que en estatal & 30 & $7.48 \%$ \\
Colegio particular & $\mathbf{2 1 5}$ & $\mathbf{5 3 . 6 2 \%}$ \\
\hline
\end{tabular}

En la Tabla 6, se observa que un porcentaje de $53.62 \%$ provienen de colegio particular y 32.92 $\%$ de colegio estatal. Es decir, a pesar de provenir de colegios particulares, donde la malla curricular es semejante al del prospecto de admisión de la universidad, para acceder a una vacante en la universidad nacional, en el caso de esta investigación, deben prepararse en una academia.

En la Tabla 7 se muestra la relación existente entre el rendimiento académico y los colegios estatales.

Tabla 7. Colegio estatal y rendimiento académico.

\begin{tabular}{ccc}
\hline Colegio estatal & Cantidad de alumnos según valoración UNI & Porcentaje \\
\hline Pésimo & 19 & $14 \%$ \\
Malo & 30 & $23 \%$ \\
Bueno & 33 & $25 \%$ \\
Aprobado & 30 & $23 \%$ \\
Excelente & $\mathbf{2 0}$ & $\mathbf{1 5 \%}$ \\
\hline
\end{tabular}


En la Tabla 7, se aprecia el rendimiento académico solo de los alumnos de colegio estatal en el ciclo 2020-1. Se observa que entre pésimo y malo se aprecia un $37 \%$; y entre bueno a excelente un 63 $\%$ considerable. Cabe recalcar que algunos se están preparando varios ciclos.
Seguidamente en la Tabla 8 se muestra la relación del rendimiento académico y los colegios particulares.

Tabla 8. Colegio particular y rendimiento académico.

\begin{tabular}{ccc}
\hline Colegio particular & Cantidad de alumnos según valoración UNI & Porcentaje \\
\hline Pésimo & 39 & $18 \%$ \\
Malo & 54 & $25 \%$ \\
Bueno & 39 & $18 \%$ \\
Aprobado & 48 & $22 \%$ \\
Excelente & 34 & $16 \%$ \\
Extraordinario & $\mathbf{1}$ & $\mathbf{0 . 4 7 \%}$ \\
\hline
\end{tabular}

En la Tabla 8, de estudiantes de colegios estatales, se observa que el rendimiento académico entre pésimo y malo nos da un 33\%, y entre bueno a extraordinario un $67 \%$ considerable. Sin embargo, en la tabla anterior, se debe considerar los años que está dedicando a la preparación.

La comparación entre colegio particular o privado y colegio estatal indica que el rendimiento académico es relativamente mejor en los estudiantes que provienen de colegios particulares, con una diferencia solo de $4 \%$. Esta información se confirma con los resultados del MINEDU (2014), en la prueba ECE-2014 que, incluso, en algunos distritos el rendimiento académico de estudiantes de colegio estatal era superiores en un porcentaje mínimo a los estudiantes de colegios privados.

\section{Rendimiento académico}

Respecto al rendimiento académico de los estudiantes preuniversitarios de Cepreuni, a febrero 2020, se encontró la siguiente información:

Tabla 9. Valoración de nota del ciclo 2020-1 según Reglamento UNI.

\begin{tabular}{ccc}
\hline Resultados & Cantidad & Frecuencia \\
\hline Pésimo & 70 & $17.46 \%$ \\
Malo & 94 & $23.44 \%$ \\
Aprobado & 85 & $21.20 \%$ \\
Bueno & 88 & $21.95 \%$ \\
Excelente & 62 & $15.46 \%$ \\
Extraordinario & 2 & $0.50 \%$ \\
Total & $\mathbf{4 0 1}$ & \\
\hline
\end{tabular}


De la Tabla 9 se observa que si bien existen 164 estudiantes en el rango de pésimo a malo, 227 estudiantes están en los rangos de rendimiento aceptable. No se debe olvidar el tiempo de preparación de la mayoría de estudiantes de este grupo. Quienes tienen mejor promedio, generalmente, tienen algunos ciclos de preparación, ya sea en Cepreuni u otra academia que se dedica a la preparación.

\section{CONCLUSIONES}

Se puede concluir que la educación básica regular estatal, no prepara para seguir estudios de educación superior porque los cinco cursos que brinda no son suficientes para responder los complejos exámenes de admisión de cada universidad nacional.

Según la edad de los estudiantes en el centro de estudios preuniversitarios, que no corresponde al ámbito formal del sistema educativo peruano, se concluye que para ocupar una vacante en la universidad nacional debe prepararse desde tercero de secundaria y, a veces, tres años más. Esto implica de parte del apoderado o padre de familia un oneroso gasto económico. Y quien no cuenta con ingresos económicos solventes, no puede ingresar a la universidad nacional.

Asimismo, la diferencia entre formarse en un colegio particular, con malla informal y semejante al balotario de los prospectos de las universidades nacionales a las que postula, y los estudiantes de colegios nacionales, según el rendimiento académico, solo es de un $4 \%$. Es decir, invertir once años o menos en colegios particulares y sin preparación en centros de estudio preuniversitario no se logra ingreso, por lo general, a universidades nacionales.

\section{REFERENCIAS}

Angulo H., P., Angulo A., P., Huamán, L., y Espino, J. (2016). Propuesta de procesos en un sistema de gestión de la calidad para la educación universitaria con formación integral y competencias profesionales. Horizonte De La Ciencia. 6 (10) , 239-259

Asencios, R. (2016). Rendimiento escolar en el Perú: Análisis secuencial de los resultados de la Evaluación Censal de Estudiantes. Perú: Banco Central de Reserva del Perú

Bravo, A. (2018). 70\% de egresados de la secundaria no va a universidades. Educación. Recuperado el 13 de Junio de 2021, de https://noticia. educacionenred.pe/2019/12/preocupanteestadistica-70-egresados-secundaria-no-vauniversidades-188524.html

Castrillón, O., Sarache, W., y Ruiz-Herrera, S. (2020). Predicción del rendimiento académico por medio de técnicas de inteligencia artificial. FormaciónUniversitaria. 13(1), 93-102.

Didriksson, A. (04 de Febrero de 2020). Panorama comparadoyregional delas tendencias decambio en la universidad actual. Recuperado el 12 de Junio de 2021, de https://www.iesalc.unesco. org/2020/02/04/webinar-sobre-tendencias-decambio-en-la-universidad-actual/

Flores, R. (2010). Acceso y Permanencia en una educación de calidad: El pensamiento crítico como una competencia transversal para la calidad dela educación. Buenos Aires, Argentina: Congreso Iberoamericano de Educación, Metas 2021

Gonzales, F. (2016). Valoración social de los cursos preuniversitarios en el sistema universitario chileno. España: Trabajo especial de grado de la Universitat Autónoma de Barcelona

Hurtado, L. (2006). Acerca de la preparación preuniversitaria. Investigación Educativa, 10 (17) , 167-174

Instituto Peruano de Economía-IPE. (2018). Dificultades en los colegios y su impacto en el rendimiento. La Industria. Recuperado el 13 de Junio de 2021, de https://www.ipe.org.pe/portal/ dificultades-en-los-colegios-y-su-impacto-enel-rendimiento/

Lamas, H. (2015). Sobre el rendimiento escolar. Propósitos y Representaciones 3(1), 313-386 
Mandelman, S., Barbot, S., y Grigorenko, E. (2016). Predicting academic performance and trajectories from a measure of successful intelligence. Learning and Individual Differences 51(1), 387-393

Maza, K. (31 de Enero de 2019). Mnedu: 6 de cada 10 jóvenes no acceden a educación superior. Recuperado el 13 de Junio de 2021, de https:// elcomercio.pe/economia/peru/minedu-6-10jovenes-acceden-educacion-superior-noticia603009-noticia/?ref=ecr

Ministerio de Educación. (2014). Resultados de la Evaluación Censal de Estudiantes 2014. Oficina de la Medición de la Calidad de los Aprendizajes. Recuperado el 13 de Junio de 2021, de http://umc. minedu.gob.pe/wp-content/uploads/2015/06/ RESULTADOS-+-FACTORES-ASOCIADOS_ ECE-4TO-EIB-2014.pdf

Ministerio de Educación.(2017).Currículo Nacional de la Educación Básica. Perú: MINEDU.

Miranda, F. (2018). Diagnóstico, teoría e intervenciones públicas para abatir el abandono escolar en la educación secundaria de segundo ciclo: aprendizajes desde América Latina. Revista Latinoamericana de Educación Comparada, 9(14), 11-30

Morazán, S. (2013). Competencias docentes y su relación con el rendimiento académico en la asignatura de matemáticas en las instituciones de educación media del municipio de Danlí. República de Honduras: Trabajo espcial de grado de la Universidad Pedagógica Nacional Francisco Morazán para optar al título de magiister en Investigación Educativa

Neira, H. (Agosto de 2020). La anomia social es el resultado de una mala educación . Recuperado el 13 de Junio de 2021, de Blog de Noticias. Perú: Edición 196.: https://inversionenlainfancia.net/ blog/entrada/noticia/4385/0
Núñez, M. (2013). Las Competencias Profesionales de estudiantes preuniversitarios en países de Iberoamérica. México: Trabajo especial de grado de la Universidad Iberoamericana

Palella Stracuzzi, S., y Martins Pestana, F. (2010). Metodología de la investigación cuantitativa. Caracas, Venezuela: FEDUPEL, Fondo Editorial de la Universidad Pedagógica Experimental Libertador

Piscoya, L. (2004). Un currículo para la antieducación. Educación 1(1), 59-66

Quiles, O., y Zaragoza, J. (2020). Educación media y superior en México: análisis teórico de la realidad actual. Revista De Educação E Humanidades, 6, $59-72$

Romero, R. (2021). Resiliencia, violencia intrafamiliar, condición sociodemográfica y su incidencia en el rendimiento académico en estudiantes preuniversitarios de ingeniería, 2020. Trabajo especial de gradode la Universidad Nacional Mayor de San Marcos

Secretaria Nacional de la Juventud. (12 de Agosto de 2019). Jóvenes representan el 25\% de la población peruana. Recuperado el 13 de Junio de 2021, de https://juventud.gob.pe/2019/08/ jovenes-representan-el-25-de-la-poblacionperuana/

Superintendencia Nacional de Educación Superior Universitaria SUNEDU. (04 de Enero de 2021). Universidades licenciadas. Recuperado el 12 de Junio de 2021, de https://www.sunedu.gob.pe/ lista-de-universidades-licenciadas/

Unesco. (2020). Perfil de país, Cuba. Siteal. Recuperado el 13 de Junio de 2021, de https:// www.siteal.iiep.unesco.org/sites/default/files/ sit_informe_pdfs/dpe_cuba_25_09_19.pdf 